\title{
Dependence of Cluster Growth on Coefficient of Restitution in a Cooling Granular Fluid
}

\author{
Subir K. Das ${ }^{1,2, *}$ and Subhajit Paul ${ }^{1,3}$ \\ 1 Theoretical Sciences Unit, Jawaharlal Nehru Centre for \\ Advanced Scientific Research, Jakkur P.O, Bangalore 560064, India \\ 2 School of Advanced Materials, Jawaharlal Nehru Centre for Advanced \\ Scientific Research, Jakkur P.O, Bangalore 560064, India and \\ ${ }^{3}$ Institut für Theoretische Physik, Universität Leipzig, Postfach 100920, D-04009, Leipzig, Germany
}

(Dated: July 10, 2020)

\begin{abstract}
Starting from configurations having homogeneous spatial density, we study kinetics in a twodimensional system of inelastically colliding hard particles, a popular model for cooling granular matter. Following an initial time period, the system exhibits a crossover to an inhomogeneous regime that is characterized by the formation and growth of particle-rich clusters. We present results on the time dependence of average mass of the clusters and that of average kinetic energy, obtained via event driven molecular dynamics simulations, for a wide range of values for the coefficient of restitution (e), by fixing the overall density of particles in the system to a constant number. The time of onset of crossover from homogeneous to the inhomogeneous regime, as is well known, strongly increases as one moves towards the elastic limit. Nevertheless, our presented results suggest that the asymptotic growth is independent of $e$, for uniform definition of cluster, onset of which has a different $e$-dependence than the onset of above mentioned crossover. In other words, not only the exponent but also the amplitude of the power-law growth, which is widely believed to be the form of the evolution, is at the most very weakly sensitive to the choice of $e$. While it is tempting to attribute this fact to the similar feature in the decay of energy, we caution that our current understanding is not matured enough to draw such a connection between cluster growth and energy decay in a meaningful manner.
\end{abstract}

PACS numbers: 47.70.Nd, 05.70.Ln, 45.70.Mg

\section{INTRODUCTION}

Granular materials [1, 2] consist of particles of varying shapes and sizes, and are very commonly observed in nature. Typical examples [1 4$]$ are powders, drugs, sacks of rice or sugar grains, packets of coffee beans, cosmic dust, etc. Thus, knowledge of the behavior of granular materials is of immense importance in many disciplines. Understanding of these, at different length and time scales, can be useful in the interpretation of the formation of planetary rings; has applications in industries like pharmaceutical, agriculture and mining; is of importance in prevention of damages due to natural processes like landslide, erosion, etc. This, however, is challenging, a reason being that often these materials share properties of both fluids and solids [1, 2].

Due to friction and inelastic collisions among par- ticles these systems continuously cool, i.e., particles loose kinetic energy, average value of which defines the granular temperature. This leads to interesting pattern formation that, for a class of systems $1,2,2,5-$ 23], resembles 24 27] coexistence of particle-rich and particle-poor clusters during vapor-liquid transitions. Over the past few decades there have been intense research activities to identify and understand the form of energy decay and cluster growth in this class of systems. Focus of the present paper is related to these.

In this context, in the original form of a popular model, to be referred to as the granular gas model (GGM) [5], energy dissipation occurs only due to inelastic collisions among hard constituents, the coefficient of restitution (e) lying between 0 and 1 . This dissipation leads to progressive parallelization of velocities of the particles and formation of clusters in the so called inhomogeneous cooling state (ICS). Like 
in kinetics of phase transitions [24, 25], here also typically one asks: How does the average mass $(m)$ of these clusters grow with time $(t)$ ? There is a reasonably fair belief that the growth is of power-law type [8, 12, 13, 15, 16]:

$$
m \sim t^{\zeta}
$$

In the ICS, the decay of average kinetic energy $(E)$ is even more widely studied aspect. It has been observed that this also follows power-law [6, 7, 14, 27]:

$$
E \sim t^{-\theta}
$$

There has been immense interest in estimation of and understanding the dependence of $\zeta$ and $\theta$ on space dimension $(d)$ and other system parameters like particle density $(\rho)$ and $e$. In this paper, $\rho$ is calculated as $N / V$, where $N$ is the number of particles and $V$ is the volume of the box. Furthermore, establishing connection between $\theta$ and $\zeta$ also remains of significant current research interest. With respect to this, while good progress has been made in $d=1$, the status is much inferior for higher dimensions. In $d=1$ there exists evidence that [6, 7, 28 , 30.

$$
m \sim 1 / E \sim t^{2 / 3},
$$

irrespective of the values of $\rho$ and $e$. In fact, GGM in this dimension is believed to be equivalent to another popular model (perhaps simpler, though extremely useful), referred to as the ballistic aggregation model (BAM) 29].

In the BAM hard spherical particles move ballistically and following a collision the partners merge to form a larger spherical entity, keeping the mass and momentum conserved. For this model Carnevale et al. 29] predicted that

$$
m \sim 1 / E \sim t^{2 d /(d+2)},
$$

implying strong, inverse relation between clustering and dissipation in all dimensions. Computer simulations, however, reported discrepancies 30 32] with this prediction in $d>1$, when packing fraction is not too high. For the BAM another theory predicts that 31, 32]

$$
2 \zeta+d \theta=2 d .
$$

At least up to $d=3$, it has been observed in simulations with different packing fractions that this hyperscaling relation is valid [16].

While no such strong and accurate connection between cluster growth and energy decay for the GGM has been established, undoubtedly the decay of the latter is the cause for the growth of the former. In this work we present results from the simulation study of this model in $d=2$ for a wide range of $e$. Our results on the growth of mass are suggestive of certain interesting universal feature. Similar feature is observed in the decay of $E$ as well. Nevertheless, we are reluctant to draw connection between the two. We cite example (from $d=1$ ) to emphasize that the relation between energy decay and growth of mass is rather complex in GGM. Thus, understanding of the observation requires further attention.

The rest of the paper is organized as follows. In section II we provide further details of the model and describe certain methods. The results are presented in section III. Finally, section IV concludes the paper with a brief summary and outlook.

\section{MODEL AND METHODS}

In the standard two-dimensional GGM [5] , that we consider here, a system consists of equisized inelastic hard discs. The velocities of colliding partners $i$ and $j$ before and after (represented with prime) an instantaneous collision are related via the equations [33]

$$
\vec{v}_{i}^{\prime}=\vec{v}_{i}-\frac{1+e}{2}\left[\hat{n} \cdot\left(\vec{v}_{i}-\vec{v}_{j}\right)\right] \hat{n},
$$

and

$$
\vec{v}_{j}^{\prime}=\vec{v}_{j}+\frac{1+e}{2}\left[\hat{n} \cdot\left(\vec{v}_{i}-\vec{v}_{j}\right)\right] \hat{n} .
$$

Here $\hat{n}$ is an unit vector aligned with the relative position of the colliding partners. Equations (6) and (7) satisfy the conservation of momentum and contain the fact that there is collisional energy dissipation by a factor $1-e^{2}$. For $e<1$, velocities of the partners become more parallel after a collision. This is qualitatively depicted in Fig. 1.

With this rule, we have performed event-driven molecular dynamics simulations [33, 34]. After every 
new collision, the task of the simulation code is to identify the partners and instant for the next collision. Between collisions these particles move ballistically, i.e., with constant velocities. Progress of time is calculated by adding the intervals between collisions 6, 7]. While this method provides the real time, in the literature dynamics of this model has been quantified by using this time as well as with respect to the number of collisions per particle [12, 13]. We do not adopt the latter here. Even though there exists linear relation [6, 15] between these two times in the ICS, this is not the case during the homogeneous period. Such discrepancy or nonuniformity between the two regimes, with respect to the connection between two different measures of time, occurs due to the following fact. At early regime, compared to the late time situation, the systems contain mostly faster moving particles. The velocity distributions [11] are different in the two regimes with large regions having velocities of particles aligned with each other at late time.

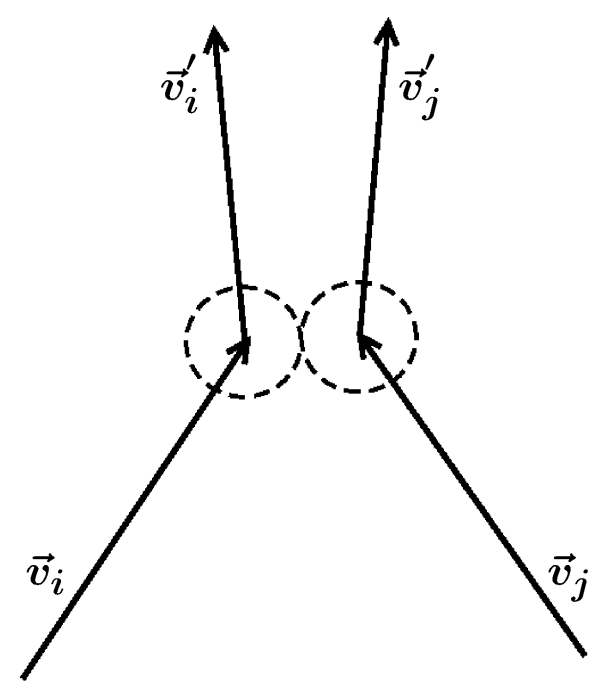

FIG. 1. Sketch of a collision event for $e<1$ in $d=2$. The particles $(i$ and $j$ ) are represented by dashed circles. The arrow-headed lines represent velocities of the particles before $\left(\vec{v}_{i}, \vec{v}_{j}\right)$ and after $\left(\vec{v}_{i}^{\prime}, \vec{v}_{j}^{\prime}\right)$ the collision. This picture is only for the purpose of qualitative demonstration and drawn without reference to a coordinate system.

At late time one encounters serious technical problem with this simulation method, particularly for low values of $e$. Often collisions remain restricted to a tiny group of neighboring particles with small relative velocities. This fact, referred to as the inelastic collapse 17], severely limits the progress of time. A method [7, 17 19] to overcome this problem considers assignment of $e=1$ for collisions corresponding to relative speed smaller than a cut-off value $\delta$. For $d>1$, however, this problem is less severe. So, most of our results were obtained by employing $\delta=0$.

All our simulations started with random initial configurations in both position and velocity, with Maxwellian distribution for the latter [33, 34]. For each set of parameter values the starting temperature was same. We have applied periodic boundary conditions in all possible directions. The quantitative results are presented after averaging over at least 10 independent initial configurations.

The clusters were identified as regions having density over a certain critical number [15, 16], chosen to be same for all values of $e$. Boundaries around the clusters were appropriately marked to facilitate the calculation of number of particles within a cluster as well as the estimation of the corresponding mean value which is the average mass $(m)$. The results for the energy correspond to the average kinetic energy, calculation of which is straight-forward.

\section{RESULTS}

In Fig. 2 we present evolution snapshots for the considered model in $d=2$. Frames from four different times of a particular run are shown. The results correspond to $\rho=0.37$ and $e=0.8$. For equisized discs of diameter unity, this value of $\rho$ corresponds to a packing fraction of approximately 0.29 . For the earliest presented time, i.e., at $t=2.5$, the particles are still homogeneously distributed over the entire system. By $t=20.8$ crossover to the ICS has started. Regions rich and poor in particles are clearly identifiable from the snapshot at $t=64.9$, average size of which has grown significantly in the last snapshot.

This growth is quantitatively depicted in Fig. 3 Here we have plotted average mass as a function of time, on a log-log scale. Data from three different values of $e$, covering a rather wide range, have been presented. In each of the cases, we have $\rho=0.37$. 


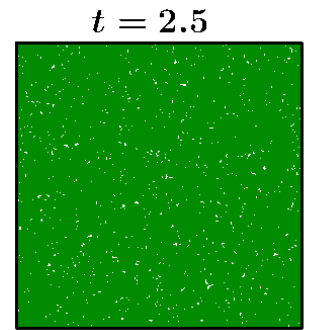

$t=64.9$

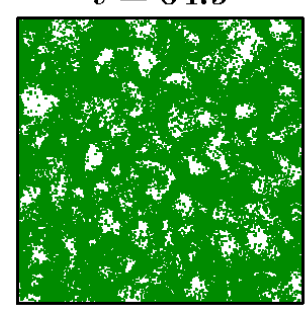

$t=20.8$

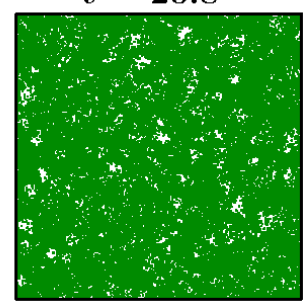

$t=435.4$

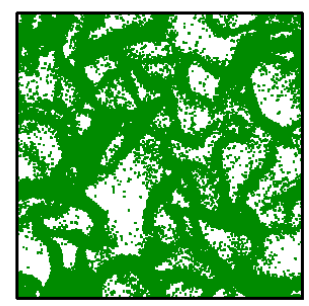

FIG. 2. Snapshots, taken during the evolution of the freely cooling granular gas in space dimension $d=2$, are presented for coefficient of restitution $e=0.8$ and overall particle density $\rho=0.37$. The location of the particles are marked. At the top of each of the frames corresponding time is mentioned.

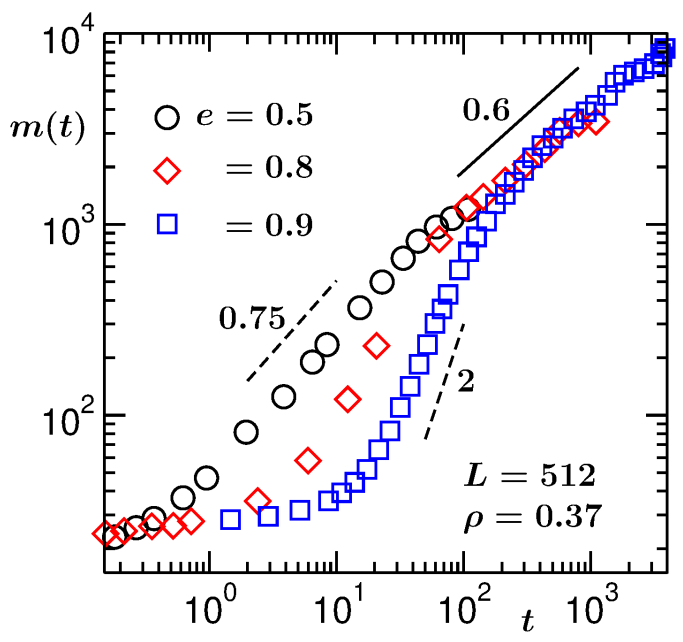

FIG. 3. The average mass of clusters $(m)$ is plotted versus time, on a log-log scale, for overall particle density $\rho=$ 0.37. Results from multiple values of $e$ are included. The solid and dashed lines represent power-laws. The values of the exponent are mentioned in appropriate places.

Given that the linear dimension ( $L$, in units of the particle diameter) of our square simulation box is 512 , the results correspond to $N=96993$. Since energy dissipation through inelastic collisions is the reason behind the clustering phenomena [5], it is expected
[12, 13] that the onset of ICS will occur earlier for smaller values of $e$. This fact can easily be appreciated from the displayed set of results. With the increase of $e$, values of $m$ remains stable at a small number, that corresponds to random, homogeneous distribution of particles, over longer periods of time.

At late time the reasonable linear appearance of the data sets on the log-log scale hints towards powerlaw behavior. The consistency of the data with the solid line suggests that 16 ]

$$
\zeta \simeq 0.6 .
$$

There has been longstanding interest in the community in estimating the exponent for this growth. Few other works [16, 29, 30], combined with these results, point to the possibility that the value of the exponent is 'practically' independent of $d, \rho$ and $e$. This contradicts both Eqs. (4) and (5). Here note that various authors [6, 16] showed that the energy decay for the present model follows Eq. (4), implying

$$
\theta=1,
$$

in $d=2$. Thus, the decay of $E$ and the growth of $m$ are not generally connected to each other via Eq. (4), clearly stating the nonequivalence between GGM and BAM in $d=2$ (and dimensions higher than that). The hyperscaling relation of Eq. (5), in a fixed dimension, has its relevance with respect to the density dependence of the two exponents. This relation, or anything analogous, also does not appear to be true for the GGM when results from other studies in $d>1$ are looked at [16].

While the above results and discussions are mostly related to strengthening of certain previously observed facts, the new interesting observation of the present study is the following. The data sets in Fig. 3 appear to overlap with each other at long times. This hints towards the fact that the scaling growths for all the $e$ values are same, not only in the exponent $\zeta$ but also in the amplitude. We repeat, onset of the crossover to the ICS gets delayed with the increase of $e$. E.g., for $e=0.5$ the onset occurs at a time less than unity $(t \simeq 0.35)$, whereas for $e=0.9$ the crossover starts at $t \simeq 3$. Nevertheless, all the data sets overlap at late time and the overlapping times appear disproportionate to those for the onset of crossover. Note that the 
ratio of the two times corresponding to the onset of crossover for largest and the smallest $e$ values is approximately 9 . On the other hand, the ratio of the times when these data sets start showing consistency with the $t^{0.6}$ behavior is approximately 3.5. This is due to sharper growth, during the crossover period, for larger $e$ value. The latter point can be clearly appreciated from Fig. 3. For the presented range of $e$ values the (approximate) power-law exponent in this regime changes from 0.75 (for $e=0.5$ ) to 2 (for $e=0.9)$. This is an interesting fact in itself.

At this point it will be useful to investigate the structural aspect in the asymptotic regime for different values of $e$. Outcome of this may lead to a more unique statement about the growth, involving mass as well as morphology.

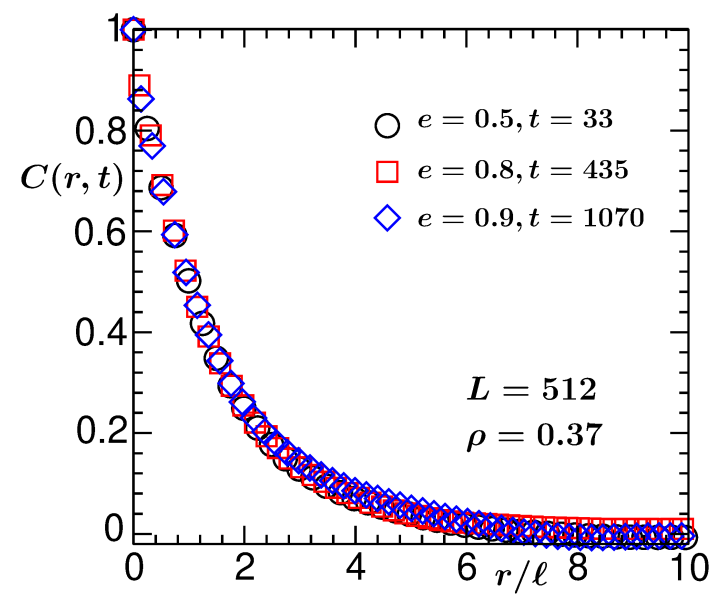

FIG. 4. Normalized two-point equal time correlation function, $C(r, t)$, is plotted versus the scaled distance $r / \ell, \ell$ being the average linear dimension of the clusters. We have shown data from three different values of $e$. In each of the cases times are chosen in such a way that the systems are in the scaling regime of growth (see Fig. 3). All data sets are for $\rho=0.37$.

In Fig. 4 we show plots of (normalized) twopoint equal time correlation function [24], $C(r, t)=$ $C_{o}(r, t) / C_{o}(0, t)$, with

$$
C_{o}(r, t)=\langle\psi(\vec{r}, t) \psi(\overrightarrow{0}, t)\rangle-\langle\psi(\vec{r}, t)\rangle\langle\psi(\overrightarrow{0}, t)\rangle,
$$

as a function of $r / \ell(t)$, where $r=|\vec{r}|$ is the scalar distance between two space points and $\ell(t)$ is the average linear dimension of the clusters at a given time $t$. In Eq. (10), $\psi$ is an appropriate order parameter
[24, 35, 36], values of which identify the particle-poor and particle-rich regions. This we have defined as

$$
\psi(\vec{r}, t)=\operatorname{sgn}\left(\rho_{\text {loc }}(\vec{r}, t)-\rho_{c}\right),
$$

where $\rho_{\text {loc }}(\vec{r}, t)$ is the local particle density at a space point $\vec{r}$ at time $t$ and $\rho_{c}$ is a cut-off density which we have set [15, 16] to 0.5 . The behavior of $C(r, t)$ provides information on the character of a structure and is commonly used in the literature of phase transitions [24, 25, 35, 36].

The average linear dimension, $\ell$, of the structure can be estimated from the decay of $C(r, t)$, say, as

$$
C(r=\ell, t)=a,
$$

where $a$ is a pre-assigned constant, having a value less than 1. In this work, however, we have estimated $\ell$ via a different route, viz., by exploiting the domain size distribution function, $P\left(\ell_{d}, t\right)$, as [26, 27]

$$
\ell=\int \ell_{d} P\left(\ell_{d}, t\right) d \ell_{d}
$$

where $\ell_{d}$ is the distance between two successive interfaces (between low and high density regions) along any Cartesian direction. There exist other methods as well in the literature [24]. Each of them provides value differing by only constant factors from the others. One needs, in this regard, to be careful that for comparative purposes unique method must be adopted.

In Fig. 4 we have included results from all three values of $e$. In each of the cases the times are chosen from the long-time power-law regime. The collapse of data, upon rescaling the distance axis by $\ell$, confirms that the structure is also similar [at least in a coarse-grained, hard-spin sense that is embedded in the calculation of $\rho_{\text {loc }}$ and definition of $\psi$ in Eq. (11)] for all values of $e$ in the asymptotic growth regime. Analogous results were presented in an earlier work [12, 13] on both growth and structure. However, in that work the range of $e$ was narrower and naturally the robustness of the phenomena, combining, on one hand, vastly different crossover times and on the other, unique asymptotic growth, could not have been captured. Also, in that work the 'real' time was not used for the quantification of growth of 'length'.

For completeness, next we demonstrate that there exists self-similarity in structure with time, a standard 
practice in studies of coarsening phenomena. For that one requires to realize superposition of data for $C(r, t)$ from different times when plotted versus $r / \ell$. In Fig. 5 we show a representative set of results, for $e=0.9$ and $\rho=0.37$. In the main frame we show direct plots, i.e., $C(r, t)$ versus $r$, from three different times. Clearly, with increasing time the decay is getting slower, implying growth in the system. In the inset we have demonstrated nice overlap of data from all the three times by scaling the distance axis by $\ell$. This confirms self-similar growth in the power-law regime of Fig. 3 for $e=0.9$. The same is true for other values of $e$ as well. However, for brevity we do not present those results.

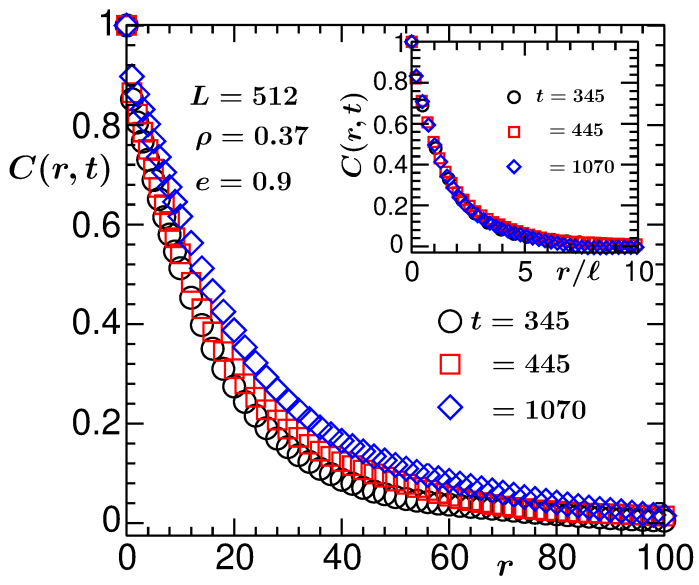

FIG. 5. Plots of $C(r, t)$ are shown versus $r$, for $e=0.9$ and $\rho=0.37$. Data from three different times are included. The inset shows same data sets but here the distance axis is scaled by $\ell$.

For possible explanation of this unique evolution, in Fig. 6 we present log-log plots of kinetic energy versus time. Again results for all three values of $e$ are shown. The late time behavior, i.e., energy decay in the ICS, is consistent with $E \sim t^{-1}$, for each of the $e$ values, which is in agreement with Eq. (4) or Eq. (9), that was also observed by other authors [6, 16]. The form of the decay prior to this is different and referred to as the Haff's cooling law [10]. It is identifiable from this figure as well that with increasing $e$ appearance of ICS gets delayed.

Here also ICS data from different $e$ values superimpose on top of each other [6]. (In the Haff's regime, for different $e$ values the deviations from each other is

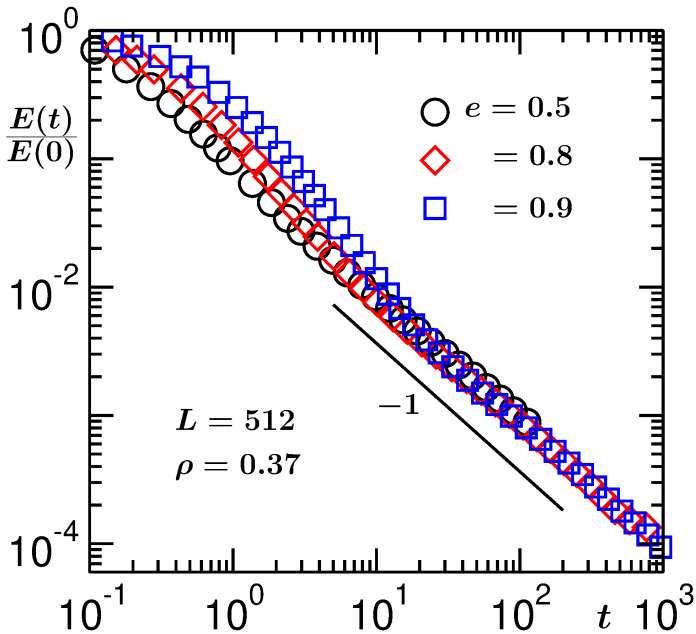

FIG. 6. Log-log plots of average kinetic energy versus time are seen. Data from three different values of $e$, for $\rho=0.37$, are shown. The solid line is a power-law. The value of the exponent is mentioned next to it.

expected.) Even though we have discussed that $E$ and $m$ cannot be connected via Eq. (4) or Eq. (5), decay of the former is the reason for growth of the latter, in the ICS, beyond doubt. Thus, one may argue that overlapping feature of mass can be explained via that in the energy data. To counter this, we would like to discuss an example from $d=1$ (cf. Ref. 28, 30]) to emphasize the fact that a connection between decay of energy and growth of mass is quite puzzling for GGM. The intention is to stress upon the fact that the explanation of the universal feature described above is not straight-forward.

In Fig. 7 we present results from $d=1$ : Part (a) contains data for decay of energy and part (b) shows the growth of mass. Results for both energy and mass are for fixed density and coefficient of restitution (see caption for these numbers). It appears that the growth of mass has frozen while the energy decay continues for much longer with the same exponent, that is consistent with the theoretical expectation of Eq. (3) or Eq. (44). Even though we have used a nonzero $\delta$ here, these results nevertheless demonstrate the presence of immense complexity in this simple model of granular matter.

In Fig. 7 even though the energy decay is clearly seen to be consistent with $\theta=2 / 3$, for the growth 

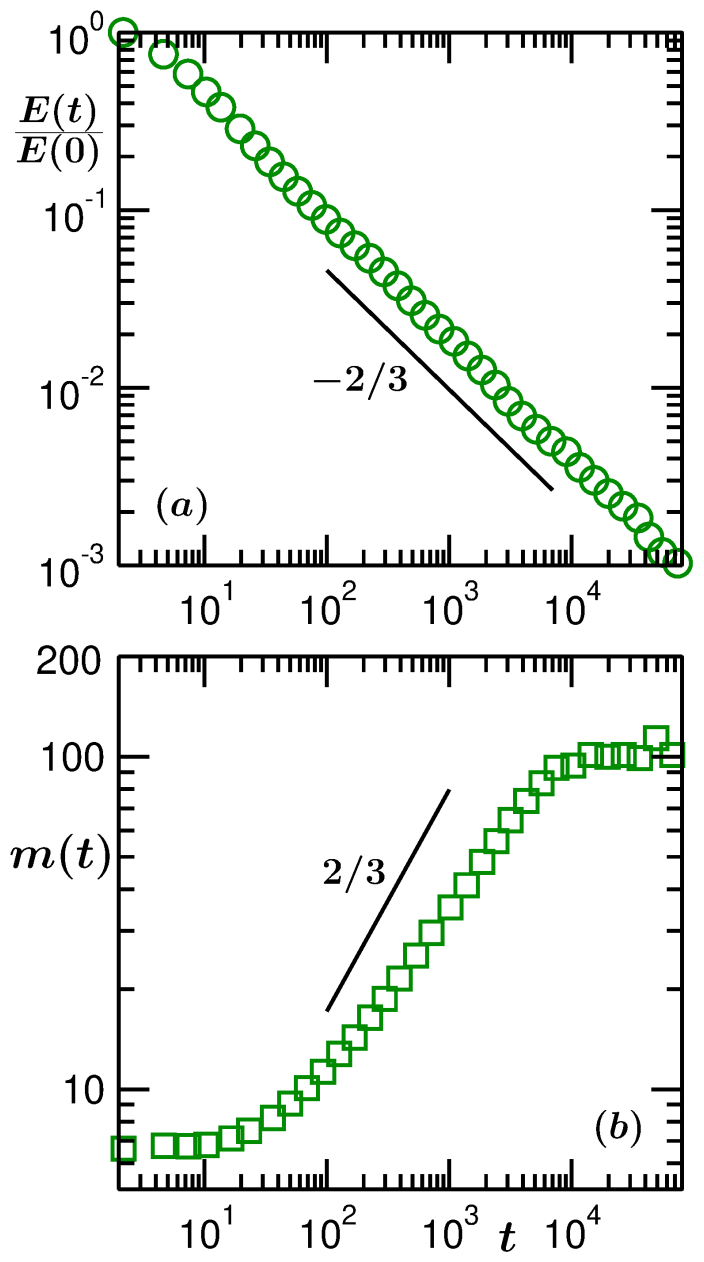

FIG. 7. Log-log plots of (a) average kinetic energy and (b) average cluster mass, versus time, for the GGM in $d=1$. All results are for $e=0.5, \rho=0.3$ and $\delta=0.005$. The solid lines represent power-laws. The exponents are mentioned next to the respective lines. The results are similar to those in Fig.1 of Phys. Rev. E 96, 012105 (2017).

of mass the value of $\zeta$ does not appear to be $2 / 3$. However, via advanced methods of analysis, including a renormalization group technique, it was confirmed [30] that $\zeta \simeq 2 / 3$.

\section{CONCLUSION}

From the event-driven molecular dynamics [33, 34] simulations we have presented results on the kinetics in a granular gas model [5]. In this model energy dissi- pation and velocity parallelization occur due to inelastic collisions among constituent particles. This leads to clustering phenomena, resembling the kinetics in a chemical system undergoing vapor-liquid transition 27. The onset of clustering strongly depends upon [12, 13] the overall particle density $(\rho)$ in the system and coefficient of restitution $(e)$.

The key result of this paper is related to the dependence of growth of mass, in the long time limit, on the coefficient of restitution. Strikingly, for a fixed overall density we observe that despite strong dependence of the onset of clustering on $e$, the asymptotic growth is same, i.e., if the character is of power-law the values of growth exponent and amplitude appear to be similar for all the presented values of $e$ that cover a rather wide range.

Of course, better statistics and more accurate analysis are necessary to put our conclusion on a concrete footing. Nevertheless, even in its current form this observation requires attention. A possible route for explanation of the phenomena could be the similar observation in the decay of energy. But we argue by providing example that the connection between energy decay and cluster growth in GGM may be more complex than realized.

Studies [37] analogous to this were performed in granular gases via direct numerical simulations of Navier-Stokes equation. The authors of this work looked at the universality in density fluctuations in the clustering phenomena with respect to the variations of different model parameters. It will be interesting to check this for GGM as well for different $e$ values. This will be useful in understanding whether the universal feature that we report here for mass should also hold for characteristic length.

More such studies are necessary to characterize universal features in granular materials. E.g., as an extension of the present work, we intend to explore a spectrum of $\rho$ and $e$ in different dimensions, in future. Even though it is not expected that growth data from different densities will collapse with each other in the inhomogeneous cooling regime, it will be interesting to check for the relevant scaling factors to obtain a master curve.

*das@jncasr.ac.in 
[1] I. S. Aranson and L. S. Tsimring, Rev. Mod. Phys. 78, 641 (2006).

[2] N. V. Brilliantov and T. Poeschel, Kinetic Theory of Granular Gases, (Oxford University Press, Oxford, 2004).

[3] N. Brilliantov, P.L. Krapivsky, A. Bodrova, F. Spahn, H. Hayakawa, V. Stadnichuk and J. Schmidt, Proc. Natl. Acad. Sci. U.S.A. 112, 9536 (2015).

[4] L. Mattson, Planet. Space Sci. 133, 107 (2016).

[5] I. Goldhirsch and G. Zanetti, Phys. Rev. Lett. 70, 1619 (1993).

[6] X. Nie, E. Ben-naim and S. Chen, Phys. Rev. Lett. 89, 204301 (2002).

[7] E. Ben-Naim, S. Y. Chen, G. D. Doolan and S. Redner, Phys. Rev. Lett. 83, 4069 (1999).

[8] S. Luding and H. J. Herrmann, Chaos 9, 673 (1999).

[9] R. Brito and M. H. Ernst, Europhys. Lett. 43, 497 (1998).

[10] P. K. Haff, J. Fluid Mech. 134, 401 (1983).

[11] A. Bodrova, A. K. Dubey, S. Puri and N. V. Brilliantov, Phys. Rev. Lett. 109, 178001 (2012).

[12] S. K. Das and S. Puri, Europhys. Lett. 61, 749 (2003).

[13] S. K. Das and S. Puri, Phys. Rev. E 68, 011302 (2003).

[14] M. Shinde, D. Das and R. Rajesh, Phys. Rev. E 79, 021303 (2003).

[15] S. Paul and S. K. Das, Europhys. Lett. 108, 66001 (2014).

[16] S. Paul and S. K. Das, Phys. Rev. E 97, 032902 (2018).

[17] S. McNamara and W. R. Young, Phys. Rev. E 50, R28 (1996).

[18] C. S. Campbell, Ann. Rev. Fluid Mech. 22, 57 (1990).

[19] S. Luding and S. McNamara, Gran. Matter 1, 113 (1998).

[20] O. Herbst, R. Cafiero, A. Zippelius, H.J. Herrmann and S. Luding, Phys. of Fluids 17, 107102 (2005).

[21] S. Takada, K. Saitoh and H. Hayakawa, Phys. Rev. E 94, 012906 (2016).

[22] S. Chen, Y. Deng, X. Nie and Y. Tu, Phys. Lett. A 269, 218 (2000).

[23] S. Miller and S. Luding, Phys. Rev. E 69, 031305 (2004).

[24] A. J. Bray, Adv. Phys. 51, 481 (2002).

[25] K. Binder, in Phase Transformation of Materials, edited by R.W. Cahn, P. Haasen and E.J. Kramer (Wiley VCH, Weinheim, 1991) Vol. 5, p. 405.

[26] S. Roy and S. K. Das, Soft Matter 9, 4178 (2013).

[27] S. Majumder and S. K. Das, Europhys. Lett. 95, 46002 (2011).

[28] M. Shinde, D. Das and R. Rajesh, Phys. Rev. Lett. 99, 234505 (2007).

[29] G. F. Carnevale, Y. Pomeau and W. R. Young, Phys. Rev. Lett. 64, 2913 (1990).

[30] S. Paul and S. K. Das, Phys. Rev. E 96, 012105 (2017).

[31] E. Trizac and J. -P. Hansen, Phys. Rev. Lett. 74, 4114 (1995).

[32] E. Trizac and J. -P. Hansen, J. Stat. Phys. 82, 1345 (1996).

[33] M. P. Allen and D. J. Tildesley, Comouter Simulation of Liquids, (Clarendon, Oxford, 1987).

[34] D. C. Rapaport, The Art of Molecular Dynamics Simulation, (Cambridge University Press, Cambridge, UK, 2004).

[35] M.E. Fisher, Rep. Prog. Phys. 30, 615 (1967).

[36] N. Goldenfeld, Lectures on Phase Transitions and Renormalization Group, (Addison-Wesley, London, 1992).

[37] M. Hummel, J. P. D. Clewett and M. C. Mazza, Europhys. Lett. 114, 10002 (2016). 